\title{
Conciencia pedagógica: Una oportunidad para aprender
}

\author{
Isabel Badilla Zamora', Anthia Ramírez García², \\ Kattia Rojas Acevedo ${ }^{3}$
}

1. Académica de la División de Educación Básica, Universidad Nacional de Costa Rica (UNA)isabel.badilla.zamora@una.cr 2. Académica de la División de Educación Básica, Universidad Nacional de Costa Rica (UNA)anthia.ramirez.garcia@una.cr 3.Académica de la División de Educación Básica, Universidad Nacional de Costa Rica, krarojasuna@gmail.com

Recibido: 27 de julio del $2015 \quad$ Corregido: 19 de octubre del 2015

Aceptado: 02 de noviembre del 2015

\section{“Educar la mente sin educar el corazón no es educación en absoluto". \\ Aristóteles}

\begin{abstract}
Resumen
La investigación aborda el tema de la conciencia pedagógica: sentir, pensar y hacer en el marco de la práctica profesional docente desarrolladas en la División de Educación Básica (DEB) de la Universidad Nacional de Costa Rica. Se concibe que el aprendizaje no sólo se produce a nivel conceptual, procedimental y actitudinal, sino que es complementado por la conciencia pedagógica. La investigación se basa en una unidad didáctica conformada por estrategias para la acción reflexión, aplicada en el marco de los cursos de Intervención Pedagógica, Proyecto Educativo en el Aula Escolar e Intervención Pedagógica desde el Aula Escolar durante el 2014. Las estrategias fueron aplicadas por el estudiantado practicante de cuyas reflexiones emergieron aspectos relacionados con los sentimientos, emociones, pasiones e incertidumbres de sus experiencias pedagógicas elementos gran importancia para lograr un aprendizaje significativo.
\end{abstract}

Palabras claves: Conciencia pedagógica: sentir, pensar y creer, formación docente, educación, identidad docente, práctica docente, responsabilidad y autorregulación.

\begin{abstract}
\section{Pedagogical awarness: an oportunity to learn}

This research addresses different topics such as ways of thoughts, feeling and doing bring about a challenge, even more so when it has to do with students who are in ways of becoming teachers. This is where a group of academics from the División de Educación Básica (DEB) from the Universidad Nacional from Costa Rica, decided to research and implement during the year 2014 a didactic unity with strategies to reflect and/or take action within the classroom.

The purpose of those strategies was reflecting and making decisions regarding the educative act in different contexts; however, the investigation showed that from the students' reflections emerge feelings, emotions, passions and uncertainties regarding their pedagogical experiences.
\end{abstract}

Key words: Pedagogical consciousness: feel, think and believe, teaching identity, education, teaching practicum, responsibility and self regulation. 


\section{INTRODUCCIÓN}

En el marco de los procesos de intervención pedagógica de las carreras de Pedagogía con Énfasis en Educación General Básica, Enseñanza del Inglés para I y II Ciclo y Educación Especial con Énfasis en Integración., las prácticas docentes han representado todo un desafío en los procesos de formación docente, por cuanto, en el ejercicio docente se espera una postura crítica y reflexiva que supere el ejercicio técnico.

A partir de la premisa citada, las investigadoras implementan una unidad didáctica con tres estrategias que generen la acción-reflexión-acción: la autoobservación, el incidente crítico y el diario de campo. Con el objetivo de conocer ¿sobre qué reflexiona el estudiantado-practicante?; así como también señalar las construcciones pedagógicas previas al nuevo accionar en el aula escolar. Sin embargo, en el proceso de socialización y revisión de las estrategias se evidenció una tendencia significativa a reflexionar en torno al sentir y pensar en su accionar durante el ejercicio docente.

Por tanto, surgió el cuestionarnos ¿qué piensan los docentes en formación y durante su proceso de práctica? y ¿Cómo lo relacionan con lo que hacen?. Las principales preguntas que nos condujeron abordar la conciencia pedagógica vinculada con la trilogía: sentirpensar-actuar; y su relación con la construcción del aprendizaje. Desde esta misma perspectiva citamos a Freire (2004) "estudiamos, aprendemos, enseñamos y conocemos con nuestro cuerpo entero. Con los sentimientos, con las emociones, con los deseos, con las dudas, con la pasión y también con la razón crítica. Jamás solo con la última" (p.8).

Es a partir de estos elementos que la formación docente construye habilidades y competencias para el aprendizaje y la enseñanza; y a su vez implica el desarrollo de una conciencia pedagógica, que se produce con base en su historia personal, la formación docente y las vivencias prácticas en el aula escolar, en ésta última es donde mayormente se visibiliza que los aprendizajes durante el proceso de práctica son permeados por las experiencias y los sentimientos.

Así, la visibilización del sentir-pensar y actuar durante el proceso de formación docente recobra un papel muy importante porque complementa los aprendizajes conceptuales y procedimentales ya que, reconocer su valor para el aprendizaje integral, es hacer visible la conciencia pedagógica, y trabajar sobre esta base, permite en primer lugar vehiculizar el sentir, pensar y actuar del docente en formación y en segundo lugar evidenciar la sensibilidad necesaria para desarrollar procesos de aprendizaje conducentes a mirar lo que se hace y se siente, pensar el por-qué cierto comportamiento en la propia práctica docente.

Desde perspectiva, es importante rescatar que la implementación de las estrategias de auto-reflexión constituye una vía de acción, reflexión y acción para los formadores de futuros docentes, en tanto les permite visualizar el desarrollo de la en términos de lo que se le pide al estudiantado y la manera como éstos reflexionan ante sus vivencias; lo cual a las investigadoras valorar el alcance más allá de lo utilitario de las estrategias. A partir de lo anterior, se valorar el sentir, pensar y actuar durante los procesos de práctica profesional supervisada, ya que vinculan el aprendizaje como parte de la construcción de los saberes; no sólo teóricos y prácticos, sino también emotivos.

\section{MARCO TEÓRICO}

La Organización de las Naciones Unidas para la Educación, la Ciencia y la Cultura (UNESCO) establece, a través de la Comisión Internacional sobre Educación para el siglo XXI presidida por Jaques Delors, cuatro pilares para guiar la formación docente: aprender a aprender, aprender a hacer, aprender a vivir juntos y finalmente aprender a ser, todos igualmente importantes.

- Aprender a aprender: el docente en su diario vivir aprende mediante sus experiencias poder comprender el mundo que lo rodea

- Aprender a hacer mejores profesionales auto-evalúa constantemente el proceder.

- Aprender a vivir juntos: el docente descubre en el estudiantado condiciones y elementos que enriquecen su aprendizaje, pero partiendo principalmente del conocerse a sí mismo, para poder reconocer al otro.

- Aprender a ser: se toman decisiones que -ayudan a crecer y mejorar como individuo .

Por lo anterior, es errado pensar que los aprendizajes producidos en los procesos de práctica docente, 
-sólo son construcciones teórico- práctico o del ámbito académico, dejando de lado otros aprendizajes como los emotivos, asociados al sentir, pensar y actuar. En ese sentido, las estrategias didácticas implementadas para la acción-reflexión-acción desvelan otros aspectos como la ética y la moral del docente en formación.

La consciencia pedagógica induce al futuro docente a incorporar su historia de vida desde su pensar y sentir fundamentalmente y en este sentido Brito (2008) comenta sobre Freire:

Precisamente está concepción de Freire acerca de la biografía del sujeto y de su lugar constituye una propuesta pedagógica que incorpora al sujeto, su historia personal, sus tradiciones culturales y populares en función de un aprendizaje apropiado desde la vivencia de los sujetos participantes en los procesos educacionales. Se convierte así el sujeto en el protagonista de su educación (p.35).

La concepción de la identidad docente se relaciona no solamente con los aprendizajes adquiridos a través de los contenidos académicos, sino también con la heterogeneidad de subjetividades sentidas, compartidas e interaccionadas con la realidad educativa en las aulas escolares para reinterpretar la realidad; que por su diversidad es compleja. Desde esta perspectiva la formación circunscribe a una comunidad donde los saberes populares sentidos, compartidos y vivenciados deben ser incorporados, ya que juegan un papel fundamental en la construcción de la identidad docente.

Al respecto, Freire, (2004) en el documento Cartas para quien pretende enseñar indica que "la programación inicial de una práctica a veces se rehace a la luz de las primeras evaluaciones que sufre la práctica. Evaluar casi siempre implica reprogramar, rectificar" (p. 14). Tal y como lo expresa Freire, el docente en formación durante las prácticas supervisadas, está en ese constante rediseño de su tarea escolar, con el propósito de mejorarla y encontrar su propio estilo en el proceso de enseñar y aprender. Es en esta etapa cuando más evaluación del proceso existe, ya que en su gran mayoría no poseen experiencia de campo, pero la autoevaluación les permite auto-regularse y tomar decisiones.

Por otra parte, Freire (2004) asegura:
Es que enseñar no existe sin el aprender, y con esto quiero decir más de lo que diría si dijese que el acto de enseñar exige la existencia de quien enseña y de quien aprende. Quiero decir que el enseñar y el aprender se van dando de manera tal que por un lado, quien enseña aprende porque reconoce un conocimiento antes aprendido y, por el otro, porque observando la manera como la curiosidad del alumno aprendiz trabaja para aprehender lo que se le está enseñando, sin lo cual no aprende, el educador se ayuda a descubrir dudas, aciertos y errores (p. 28).

Con base en lo anterior, se considera que el educador en formación aprende, en primera instancia como sujeto socio-cultural, aprende haciendo (enseñando), pero también aprende al sentir y experimentar emociones. Asimismo, la práctica docente ofrece la experiencia que el futuro formador necesita, no solo en cuanto a lo conceptual, sino que a todas aquellas acciones que conlleva el acto y proceso de enseñar. A lo que Freire llama preparación y capacitación permanente para rehacer su saber y sentir, basándose en la auto observación y la experiencia adquirida con el diario vivir para actuar y hacer una nueva lectura del mundo.

Las emociones consideradas por las investigadoras como parte de los saberes adquiridos y experimentados nos inducen a considerar su revisión y relación con el aprendizaje, máxime cuando este proceso ocurre en un espacio sensitivo cargado de pensamientos y sentimientos de todo tipo.

Al respecto, Maturana (2001) considera que "si queremos entender las acciones humanas no tenemos que negar el movimiento o el acto como una operación particular, sino a la emoción que lo posibilita" (p.65).

Todas las acciones nacen de una emoción y ésta es considerada como la conciencia pedagógica del docente en formación, y una de las bases fundamentales para el accionar que va más allá de lo técnico-práctico del proceso de enseñanza aprendizaje mediado por el docente. Las manifestaciones de las emociones se evidencian lógicamente en el lenguaje utilizado para la reflexión de sus experiencias prácticas, pero no se debe obviar que ese lenguaje también es una síntesis de sus saberes y un producto de la lectura reflexiva de la realidad, con el propósito de construir propuestas pedagógicas más contestatarias y pertinentes. 
Se desprende de lo anterior, que lo importante es rescatar el lenguaje del estudiantado, ya que como dice Maturana, (2001) "si no estamos en el lenguaje no hay reflexión, no hay discurso, no decimos nada, simplemente somos sin serlo hasta que reflexionamos sobre el ser" (p.25). Reflexiona así sobre el ser a través del lenguaje que unido a las emociones permite al docente en formación darse cuenta de la necesidad e importancia de la reflexión para el saber, pero también, para el saber actuar. Al respecto Maturana, (2001) agrega "no es la razón la que nos lleva a la acción sino la emoción" (p.13). Cada vez que el estudiantado reconoce que ciertas cosas son obvias o son así en la escuela, lleva a considerar, que ellos pueden estar racionalizando los actos escolares desde dos dimensiones, desde la razón o desde su conciencia pedagógica: piensan, sienten y actúan.

Bajo este lineamiento el lenguaje vehiculiza esas emociones y también le da sentido a la realidad observada. Igualmente, acompaña la construcción de la toma de la conciencia pedagógica y su diversidad de usos, por lo que es necesario identificar en este estudio los ejes de significación para su análisis e interpretación.

Los ejes se recortan y contribuyen a un nuevo ordenamiento de las concepciones; son "puntos de almohadillado o nudos de significación" compartidos por un conjunto de docentes. En este sentido representan lo común, lo que los define y los diferencia de otros grupos de docentes (Shön, 1998, pp.31-41).

Válido entonces señalar el papel que juega el lenguaje en el aprendizaje a través de las emociones y en la construcción de la identidad docente

El eje tipifica al grupo y subyace a la formulación de las concepciones individuales. Tiene carácter histórico, sintetiza lo individual y lo social y está asociado a los procesos de constitución de la identidad.

El eje al evidenciar lo típico, el referente identificatorio común devela relaciones de proximidad y distancia entre las concepciones de cada sujeto con dicho referente, así como también las que pueden existir entre los ejes tipificadores de cada grupo (Shön, 1998 pp.31-41).

Entre la diversidad de lenguajes se encuentra lo diverso y lo común, en este contexto el proceso de aprendizaje se deconstruye constantemente para conformar los saberes más subjetivos y creativos; además brinda la posibilidad al estudiantado en formación de utilizar sus saberes al completo para la toma de decisiones.

Asimismo, es necesario explorar la inteligencia emocional, según los investigadores Mayer y Salovey (citados por Ostrovsky, 2006) "consiste en la habilidad de manejar los sentimientos y las emociones, discriminar entre ellos y utilizar estos conocimientos para dirigir los propios pensamientos y acciones" (p. 168). Este concepto ha tenido constantes reformulaciones, sin embargo hemos decidido retomarlo por toda su vigencia y aplicabilidad en los procesos de formación.

Goleman (citado por Ostrovsky, 2006), establece que la inteligencia emocional se caracteriza por conocer sus propias emociones y manejarlas, motivarse a sí mismo, reconocer las emociones de los demás y establecer relaciones. Además, Goleman (citado por Ostrovsky, 2006, p. 107), nos habla del concepto de conciencia de uno mismo, "la capacidad de percibir los estados internos propios, la auto reflexión acerca de la experiencia y las emociones propias, incluso en momentos problemáticos y difíciles de afrontar (por ejemplo, restringir la ira en el momento en que la estamos viviendo)".

Se dice que las personas que logran desarrollar sus habilidades emocionales cuentan con más probabilidades de sentirse satisfechas, logran ser eficaces en su vida y dominar los hábitos mentales que favorecen su propia productividad. En concordancia con lo antes expuesto, los sentires y pensares del docente en formación juegan un papel fundamental en la construcción del aprendizaje significativo y de la apropiación de sus propias emociones para leer la realidad en la que está inmerso, procesos fundamentales para la transformación propia y del contexto escolar.

\section{Formación docente}

Los planes de estudios de las carreras de Pedagogía de la División de Educación Básica, asumen epistemológicamente la práctica docente como una construcción de conocimiento basada en una relación práctica-teoría-práctica. Se parte del supuesto de que la intervención presenta tres momentos, uno en que el alumno observa y sistematiza el quehacer docente en un grupo 
escolar: un segundo momento en que el estudiante no sólo sistematiza, sino que inicia ciertas labores a cargo de un grupo específico en un centro educativo: finalmente un momento en el cual el estudiante toma el control de un grupo de niños en un centro educativo durante al menos un día laboral (Bejarano, Flores, et al., 2010).

A partir del accionar docente el aprendizaje no solo se va configurando, sino que la experiencia de aula escolar le permite sacar al estudiantado todas las emociones experimentadas en el aula escolar para leer la realidad no solo desde la teoría, sino desde sus sentires y pensares en la búsqueda constante de respuestas.

Asimismo, la práctica docente se convierte en un espacio de investigación permanente y una oportunidad para perfilar su práctica profesional. Parafraseando a Sanjurjo (2009), el proceso reflexivo es aquel que permite darnos cuenta y dar cuenta de nuestras propias acciones y de la diversidad de condicionantes que las determinan. La reflexión se constituye en un puente entre la teoría y la práctica, entre el pensamiento y la acción, entre los materiales curriculares, metodología y los problemas que nos plantea la realidad. Para enfatizar en los procesos de reflexión realizados por el estudiantado, durante la práctica docente se refleja que toman en cuenta los procesos pedagógicos y que existe una tendencia a sistematizar situaciones desde la esfera afectiva-emocional de los procesos de enseñanza y aprendizaje.

\section{MARCO METODOLÓGICO}

El proceso de investigación empleado fue el enfoque cualitativo porque se recogen, analizan y categorizan algunas expresiones, denominadas en el estudio frases extraídas de las reflexiones del estudiantado. Se parte de una pregunta abierta por parte de las investigadoras ¿qué piensan, sienten y creen las estudiantes y los estudiantes durante su proceso de práctica? Se aplicó una unidad didáctica conformada por tres estrategias para la acción-reflexión durante el proceso de práctica: el diario de campo, la auto-observación y el incidente crítico.

Se realiza un estudio de tipo exploratorio que intenta recoger el sentir, el pensar y el actuar debido a su incidencia en la fase exploratoria. La población participante, seleccionada al azar, se corresponde con $30 \%$ del estudiantado matriculado en cada uno de los cursos de Intervención Pedagógica desde el Aula Escolar, en la carrera Pedagogía con Énfasis en Educación Básica, en Intervención Pedagógica en la carrera de Educación Especial con Énfasis en Integración y en el Proyecto Educativo en el Aula Escolar en la Enseñanza del Inglés para I y II ciclo.

Para el desarrollo de la investigación se definen tres momentos, a saber:

- Primer momento: el estudiantado practicante realiza procesos de intervención pedagógica en la realidad de aula, reflexiona sobre la experiencia utilizando una estrategia para la acción-reflexiónacción y procede a la sistematización

- Segundo momento: las profesoras del curso y las investigadoras, generan espacio en el aula universitaria para la socialización de la estrategia para la acción-reflexión-acción, asimismo se recoge el documento en físico. En este mismo proceso, las investigadoras realizan la lectura, análisis y sistematización de las reflexiones, sin omitir que se valora la reflexión crítica del proceso en las dimensiones conceptuales, procedimentales y emotivas, además de los diferentes momentos de la reflexión: antes, durante o después de una experiencia de intervención pedagógica. Por último, las investigadoras realizan una selección de algunas reflexiones registradas por el estudiantado en sus portafolios y referidas al sentir, pensar y actuar de las tres carreras, así como, criterios de reiteración relacionada con la temática y de conveniencia para las investigadoras

- Tercer momento: se sistematizan las reflexiones según las siguientes categorías:

Sentir: el acto de percibir una emoción o sensación por parte del docente en formación y proveniente de un acto importante o novedoso en el contexto escolar o espacio pedagógico.

Pensar: es una actividad realizada por el docente en formación en los procesos de intervención pedagógica cuando se forma ideas y representaciones de la realidad en su mente que se vuelven a significar mediante estrategias dialógicas con el fin de relacionarse unas con otras.

Creer: es un ideal que tiene el estudiantado en formación como posible o bien probable. 
Actuar: el acto realizado por el docente en formación producto de un proceso que implica pensar y creer, teniendo en cuenta diversos elementos o juicios a la hora de interpretar una situación específica y que se desarrolla mediante una acción durante la enseñanza.

Los conceptos anteriores constituyen una de las bases referenciales para la sistematización de la información.

\section{DISCUSIÓN DE RESULTADOS}

Las frases que se analizan son tomadas de las estrategias de acción-reflexión-acción: la auto-observación, el incidente crítico y el diario del profesor, reflejan lo que se denomina la conciencia pedagógica.

En la tabla 1, se organizan las reflexiones referidas al sentir de los y las estudiantes de práctica, de la carrera de Pedagogía con Énfasis en I y II ciclo de la Educación General Básica, de Enseñanza del Inglés para I y II Ciclo y de Educación Especial con Énfasis en Integración, durante el año lectivo 2014.

\section{CUADRO 1}

\section{FRASES}

El evitar enojarme me ayudó a pensar, sé que este sentimiento genera más tensión, sé que molesté, traté de controlarlo y evitar que se notara.

\begin{abstract}
Al llegar a mi casa sentí la necesidad de sentarme a pensar en todo lo que me había sucedido durante la jornada, cómo en un lapso de tan pocas horas fui capaz de sentir tantas cosas a la vez, cólera, alegría, satisfacción, y demás, cómo seguía la misma situación en pie, cómo el comportamiento de los niños y niñas era tan variado, cómo se aburrían y luego estaban súper activos, cómo este proceso tan cansado se convierte en agradable y divertido gracias a los chicos y chicas, que al lado de estos y estas los momentos de cólera y frustración se borran cada vez que sonríen y cada vez que me hacen sentir orgullosa por el avance en el aprendizaje que dan día a día en su proceso de enseñanza-aprendizaje.
\end{abstract}

El trato hacia los estudiantes, el ser cariñosa me ha ayudado a acercarme a ellos. Cada vez conozco a los estudiantes un poco más, lo que me permite estar más atenta y anticipar algunas eventualidades.

Creo que las relaciones tanto intra como interpersonales son un área fuerte, porque yo me llevo bien tanto con la docente del grupo como con las estudiantes y los estudiantes. El hecho de abrazarlos, reír con ellos, apoyarlos, y también poner orden cuando tengo que hacerlo ha ayudado mucho a fortalecer el vínculo.

Sentí la emoción de que las actividades me dieran resultados y me motivaran a seguir con Incidente crítico la práctica.

\section{Análisis}

Las frases reflejan que efectivamente el estudiantado expresa una variada gama de sentimientos y desde este punto de vista se considera que la práctica docente es una oportunidad para que aprendan a identificar sus propios sentimientos, encausarlos de forma positiva y actuar buscando la asertividad o reconociendo una necesidad.

\section{ESTRATEGIAS}

Incidente crítico

Diario del profesor

Incidente crítico

Auto-observación

Fuente: Elaboración propia a partir de las estrategias de acción-reflexión-acción.

Las emociones llevan al estudiantado practicante a tomar acción, desde su propia subjetividad y dentro de su comunidad de aprendientes.

En la tabla 2, se organizan las reflexiones referidas al pensar de los y las estudiantes de práctica, de la carrera de Pedagogía con Énfasis en I y II ciclo de la Educación General Básica, de la Enseñanza del Inglés 
para I y II Ciclo y de Educación Especial con Énfasis en Integración, ciclo 2014.

\section{Análisis:}

El proceso de pensar desde las emociones experimentadas y vividas durante el proceso de práctica, permitió al estudiantado construir ideas sobre todos los actores y factores involucrados en el proceso de enseñanza aprendizaje, además conocer y entender las múltiples interrelaciones entre el sentir, pensar y actuar experimentado por el estudiantado durante los procesos de practica en el aula escolar, y esto es lo que influye en gran medida en esta interpretación de la realidad.

\section{CUADRO 2}

\section{FRASES}

Pienso que la situación presentada pudo ser evitada si se fomentara la práctica de valores y normas de cortesía dentro de la clase, considero que en general los niños de la escuela necesitan aprender más a cómo relacionarse con sus compañeros y a respetarse entre ellos.

Pienso que la maestra lo peor que puede hacer es reaccionar con más violencia ante un com- Incidente crítico portamiento hostil.

Existen muchos detalles que se nos escapan, y muchas veces idealizamos o soñamos con un mundo diferente que lamentablemente no es tan fácil de lograr teniendo tantos factores en contra, existen muchos deseos de cambiar y de hacer cosas diferentes para los niños, queremos mejorar el proceso de enseñanza que existe que es solo de pequeños receptores.

Para los niños las actividades deben ser más dinámicas o lúdicas que llamen la atención y el interés de los mismos. Si bien la actividad de presentación delante de los demás no es mala, la repetición constante de la misma hace perder el interés

Ahora que pienso en la situación con el niño, no puedo imaginar que hubiera hecho si no hubiera llegado el profesor a encargarse del incidente, me sentí muy asustada al ver el comportamiento del niño. Lo único que sí sé, es que jamás me hubiera comportado como el profesor lo hizo gritándole de esa forma, pienso que eso empeoró la situación sabiendo los antecedentes del niño.

Lo que observe hoy en la escuela me hizo recordar cómo me sentí cuando mi niña me gritaba y como esto me afectaba y reflexioné que es algo que nunca trataré de hacer.

Como lo dice la reflexión que adjunte, no debemos dar órdenes gritando, creo que la mejor manera es pidiendo simplemente las cosas y no poniéndolos en ridículo ante los demás. Hablar con ellos al final de la clase individualmente sería la mejor decisión y tratar de conocerlos para saber sus intereses.

Durante el desarrollo de las lecciones dentro del proceso de la IP aprendí que algo de gran importancia es reflexionar sobre lo bueno o malo que se nos presenta tanto dentro del aula como fuera con los estudiantes, docentes, compañeros y profesores, puesto que es lo que nos permite crecer como personas y profesionales.

Como docente es la forma de tomar las situaciones imprevistas que suceden en los servicios de educación especial, tal vez no se tiene la experiencia para resolver totalmente el problema, pero se atiende y se hace lo posible para solucionarlo o para buscarle alguna salida a la situación.

Todo el camino de regreso a mi casa, venía pensando, ¿qué fue lo que hice mal?, ¿por qué esto no me funcionó?, y ¿si me vuelve a pasar, qué hago? Fue un camino muy largo y poco motivador.

Tener la humildad para aceptar que cometo errores y que los tengo que corregir también Incidente crítico siento que me ha ayudado mucho.

Fuente: Elaboración propia a partir de las estrategias de acción-reflexión-acción.

Auto-observación

Auto-observación

Diario del profesor

Diario del profesor

Incidente crítico

Diario del profesor

Auto-observación

Incidente crítico

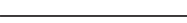


El pensar es el motor que impulsa las acciones del docente en formación y, en este caso en particular, las reflexiones realizadas por ellos evidencian la pertinencia de realzar la emoción como un eje en el aprendizaje.

En la tabla 3, se organizan las reflexiones referidas al creer de los y las estudiantes de práctica, de la carrera de Pedagogía con Énfasis en I y II ciclo de la Educación General Básica, de la Enseñanza del Inglés para I y II Ciclo y de Educación Especial con Énfasis en Integración, ciclo 2014.

\section{Análisis}

La capacidad de creer a partir de las experiencias prácticas, permite experimentar una gama de posibilidades de enseñanza aprendizaje. El creer como un proceso de construcción posible o probable del

\section{CUADRO 3}

\section{FRASES}

Pero después de mucho pensar y analizar, llegué a trascender más allá de lo que yo sentía, llegué a pensar en lo que sentían los chicos y chicas en ese momento, ¿qué cosas les pasaban por la cabeza para comportase así?, ¿algo les estaba causando ese comportamiento?, ¿sería que mi clase era muy aburrida?. ¿el tema les interesaría o no?, ¿sería que traían problemas de la casa, que los alteraba, y que esa forma de comportarse era como un escape a esa frustración?

Siempre tenemos algo que mejorar y tenemos la oportunidad de hacerlo mejor.

A nivel personal, yo crecí en Hatillo en uno de esos barrios problemáticos. Y por experiencia conozco los ambientes en que se desarrollan estos niños, en este caso los niños vienen de familias disfuncionales, donde es muy posible que vivan en una cotidiana violencia. Por lo cual no me extraña que desarrollen los mismos ejemplos en la escuela.

Creo que de haber llegado un poco más temprano, las cosas me hubieran salido mucho mejor, ya Incidente crítico que no tuve tiempo de prepararme antes de la clase ni calmar mis nervios.

Creo que una buena forma de haber resuelto la situación era tranquilizando al niño, hablarle y ha- Incidente crítico cerle ver que estuvo mal su comportamiento. Sin embargo no sé si hubiera sido capaz de reaccionar de esa forma durante la rabieta.

Creo que el error está en considerar que lo más importante en la escuela lo más importante son los Auto-observación contenidos y las pruebas escritas.

Sentarme a reflexionar con los estudiantes acerca de las actitudes presentadas. Les explicaría que Auto-observación no deben actuar de esta manera.

Me di cuenta de que era importante creer en mí para lograr aprendizajes.

Creo que educar con amor es la clave del aprendizaje.

Creo que un docente puede o no marcar a un estudiante para toda la vida.

El uso del material didáctico es clave para el aprendizaje de una segunda lengua.

Fuente: Elaboración propia a partir de las Estrategias de acción-reflexión-acción.
Incidente crítico

Diario del profesor

Diario del profesor

Auto-observación

Incidente crítico

Incidente crítico 
conocimiento le abre las puertas al estudiantado para que logre adquiera un conocimiento nuevo que le va a permitir seguir creciendo mediante el ensayo y el error, este último proceso termina dándole la posibilidad de atreverse transformar para aprender.

Entonces el creer como un componente del aprendizaje brinda la posibilidad de estar preparado para actuar asertivamente ante situaciones diversas.

En la tabla 4, se organizan las reflexiones referidas al actuar de los y las estudiantes de práctica, de la carrera de Pedagogía con Énfasis en I y II ciclo de la Educación General Básica, de la Enseñanza del Inglés para I y II Ciclo y de Educación Especial con Énfasis en Integración, ciclo 2014.

\section{Análisis}

El actuar está unido a la subjetividad ya que motiva una serie de juicios y valores para interpretar situaciones que el futuro docente considera valiosas en el -espacio pedagógico. El actuar es una decisión que

\section{CUADRO 4}

\section{FRASES}

Desde la primer lección que trabajé con este grupo se habló de la importancia y la práctica de los valores, en especial del respeto, y les aclaré que todos éramos como una familia donde nos teníamos que querer y ayudar. Traté de incentivar este ideal a lo largo de todas las lecciones. Pero hay situaciones donde los niños por su instinto y niñez no piensan en las consecuencias de sus actos. Pero no por esta razón permití que la situación pasara a más.

Mi mayor deseo era plasmar en los niños y las niñas el respeto al prójimo y al medio que los rodea, fomentar en los niños y las niñas valores, responsables y conscientes de la realidad en que viven y seguros de sí mismos; personas de bien social, integrales, capaces de usar su inteligencia, voluntad y razón para resolver los problemas de una manera pacífica.

Un apoyo fundamental sería trabajar en conjunto con los padres de familia, basándonos en las Incidente crítico mismas herramientas o estrategias en donde ellos trabajen el manejo de límites desde sus hogares.

Hacer estrategias innovadoras, que las estudiantes y los estudiantes se sientan motivados a aprender, que se sientan felices de ir a la escuela y de ver a la maestra. Enseñar a cuidar la naturaleza, a reciclar, a utilizar la tecnología, son métodos que ayudan a una innovación.

Sólo la práctica me puede dar la confianza que necesito.

Incidente crítico

Me di cuenta de que si no me comprometía, no pongo en práctica y no me arriesgo, no conseguiré Diario del profesor cambios en los estudiantes.

Al realizar el taller con los estudiantes me di cuenta del valor de aprender haciendo y poder re- Auto-observación flexionar en la importancia del compartir para lograr motivación.

Fuente: Elaboración propia a partir de las estrategias de acción-reflexión-acción.

es coherente con lo que se piensa y se cree, lo que se traduce dentro del proceso de aprendizaje en dar respuestas a una necesidad encontrada en el proceso. Asimismo, se brinda la posibilidad de aportar al contexto aspectos de su propia identidad y que guían su accionar en la toma de decisiones.

En síntesis, cuando el estudiantado logra valorar su propia práctica es posible -reprogramar, rectificar y resignificar, ya que el arte de enseñar es irrealizable sin aprender a identificar su valor y tomar consciencia del porqué llevan a cabo ciertas acciones.

\section{CONCLUSIONES}

Los procesos de práctica profesional supervisada son considerados como una construcción basada en una relación práctica-teoría práctica, sin embargo, se 
considera que el concepto debe trascender e incluir el desarrollo de la conciencia pedagógica que se posibilita.

La conciencia pedagógica del docente en formación puede llegar a ser una estrategia metodológica en la medida en que se presente la posibilidad de retomar el sentir, el pensar y el actuar del estudiantado en formación. No obstante es un elemento del aprendizaje que muchas veces invisibilizado.

La heterogeneidad en la conciencia pedagógica: debe constituir un eje articulador en la formación de la identidad docente.

Las estrategias reflexivas permiten visualizar las historias de vida de los docentes en formación a través del lenguaje utilizado. Evidencian la necesidad de retomar los aspectos emotivos ya que estos constituyen una base para el accionar en el aula.

Las estrategias utilizadas para el auto-análisis del desempeño durante la práctica supervisada también dieron la oportunidad de compartir el aspecto humano (sentir, pensar y actuar) fundamental para poder aprender, ser, hacer y definitivamente convivir.

Cuando se forma parte de un proceso educativo o se involucra con plena consciencia, no solo del contexto escolar, sino también del papel activo en él, la transformación del proceso es generalmente significativa para el aprendiz.

Es necesario incluir en los procesos formativos la reflexión del pensar, el sentir y el creer, como medio para alcanzar el cambio posible, pertinente y sostenible.

El aprendizaje para que sea significativo debe tomar en cuenta el sentir, pensar, creer y valorar que posee el estudiantado como el motor que impulsa el accionar del docente. La construcción de saberes del docente en formación también se provoca a partir del compartir sentimientos y experiencias. El verdadero aprendizaje es el que perdura a través del tiempo y toma en cuenta lo que el estudiantado piensa y siente, ya que así se convierte en el sujeto protagonista de su proceso educativo.

\section{REFERENCIAS}

Bejarano, S., Flores, L., Herrera, A., Jiménez, R. y León, H. (2010). Plan de estudios de la carrera de licenciatura de pedagogía en I y II ciclos de la educación general básica. Heredia, Costa Rica: División de Educación Básica. Material inédito.

Brito, Z. (2008). Educación popular, cultura e identidad desde la perspectiva de Paulo Freire. En Freire, P. Contribuciones para la pedagogía. Godotti, M, Gómez, M, Mafra, J y Fernández de Alencar, A. CLACSO. Buenos Aires, Argentina. Disponible en: http://bibliotecavirtual. clacso.org.ar/ar/libros/campus/freire/06Brito.pdf

De la Cruz, M. (1998). La enseñanza: ejes y concepciones. Estudios pedagógico. (Valdivia), (24). Disponible en: http://www.scielo.cl/scielo.php?script=sci_arttext\&pid $=$ S0718-07051998000100002\&lng=es\&tlng=es. 10.4067/ S0718-07051998000100002.

Delors, J. (1994): Los cuatro pilares de la educación" en La educación encierra un tesoro. El Correo de la UNESCO. Disponible en: http://es.scribd.com/doc/26440584/ Delors-Jaques-Los-Cuatro-Pilares

Freire, P. (2004). Cartas a quien pretende enseñar. Buenos Aires: Siglo XXI. Editores, Argentina.

Maturana, H. (2001). Emociones y Lenguaje en educación y Política. Disponible en: http://ir.nmu.org.ua/bitstream/ handle/123456789/128742/28b9199a65f4847eda805fc3f bc40de2.pdf?sequence $=1$

Ostrovsky, G. (2006). Cómo construir competencias en los niños y desarrollar su talento: para padres y educadores. Editorial: Editora Cultural Internacional LTDA

Sanjurjo, L. O. (Coord.). (2009). Los dispositivos para la formación en las prácticas Profesionales. Rosario: Homo Sapiens.

Shön, D.A. (1998). Estudios Pedagógicos, N²4. Disponible en: http://www.scielo.cl/scielo.php?pid=S0718-051998000 100002\&script=sci_arttext 